\title{
Crises epilépticas no período neonatal: análise descritiva de uma população hospitalar
}

\author{
Neonatal epileptic seizures: descriptive analysis in a hospital population
}

\author{
Soniza Vieira Alves-Leon', leda Lucia P. Bravo², Ana Maria Pontes ${ }^{3}$, Gustavo Medeiros de A. Figueira4, \\ Isabella D'Andrea Meira ${ }^{5}$, Luiz Claudio S. Thuler ${ }^{6}$
}

\section{RESUMO}

Objetivo: Investigar a incidência e a letalidade de crises epilépticas neonatais e as condições associadas à sua presença.

Métodos: Estudo retrospectivo observacional de base hospitalar com crianças nascidas de janeiro de 1995 a dezembro de 2004 acompanhadas por quatro semanas após o parto. Analisaram-se os dados referentes aos períodos pré-natal, intraparto e neonatal.

Resultados: Foram identificados 6.600 nascidos vivos de 6.483 partos, encontrando-se 61 casos incidentes de crises epilépticas neonatais $(0,9 \%)$. A primeira crise ocorreu até 12 horas após o parto em 45,3\% dos neonatos. Das mães analisadas, 32,8\% apresentaram síndromes hipertensivas. Entre os 61 pacientes, 91,8\% eram conceptos únicos e $4,9 \%$ eram primeiro e segundo gemelares. O peso foi menor que $2500 \mathrm{~g}$ em $50,8 \%$ e a restrição do crescimento intrauterino ocorreu em 25,9\% das gestações analisadas. Obervaram-se: hipoglicemia neonatal $(56,5 \%)$, icterícia neonatal $(52,1 \%)$ e asfixia perinatal $(70,7 \%)$. Desenvolveram sepse bacteriana 32 crianças e 17 apresentaram síndrome de aspiração meconial ao nascer. O desequilíbrio ácido-básico ocorreu em $68,1 \%$ ao longo de 28 dias pós-parto. A letalidade foi de 47,4\%, sendo mais frequente no sexo masculino $(65,6 \%)$ e em filhos de mães afro-descendentes $(67,2 \%)$.

Conclusões: A incidência de crises epilépticas no período neonatal identificada neste estudo foi três a quatro vezes superior à incidência relatada em hospitais de países desenvolvidos, embora as características dos casos fossem semelhantes. A letalidade foi de $47,4 \%$ e a asfixia grave foi a condição patológica intraparto mais frequente.

Palavras-chave: recém-nascido; convulsões; incidência; asfixia neonatal.

\section{ABSTRACT}

Objective: To investigate their incidence and lethality of neonatal epileptic seizures and their associated conditions.

Methods: A retrospective observational hospital-based study was carried out in infants born between January 1995 and December 2004. The infants were followed up for four weeks after birth. Data on the prenatal, intrapartum and neonatal periods were analyzed.

Results: Among 6,483 deliveries resulting in 6,600 liveborn infants, 61 cases of neonatal epileptic seizures were identified $(0.92 \%)$. The first seizure occurred within 12 hours after birth in $45.3 \%$ of the newborn infants. Hypertensive syndromes were present in $32.8 \%$ of mothers and intrauterine growth restriction occurred in $25.9 \%$ of the pregnancies. Of the 61 newborn infants, $91.8 \%$ were single births and $4.9 \%$ were first or second twins. In $50.8 \%$ of the infants, birthweight was below 2500g. Associated conditions were: hypoglycemia in $56.5 \%$, jaundice in $52.1 \%$ and perinatal asphyxia in $70.7 \%$. Thirty-two infants developed
Instituição: Universidade Federal do Estado do Rio de Janeiro (Unirio), Rio de Janeiro, RJ, Brasil

${ }^{1}$ Doutora em Medicina pela UFRJ. Livre Docente e Professora Associada da Unirio. Médica do Hospital Universitário Clementino Fraga Filho da UFRJ, Rio de Janeiro, RJ, Brasil

${ }^{2}$ Mestre em Neurociências pela Unirio, Rio de Janeiro, RJ, Brasil

${ }^{3}$ Especialista em Pediatria pela Unirio, Rio de Janeiro, RJ, Brasil

${ }^{4}$ Médico, Mestrando do Programa de Pós-graduação em Neurologia da Unirio, Rio de Janeiro, RJ, Brasil

${ }^{5}$ Doutora em Neurologia pela UFRJ. Professor titular da Escola Superior de Ensino Helena Antipoff, Rio de Janeiro, RJ, Brasil
${ }^{6}$ Doutor em Medicina pela UFRJ. Professor adjunto do Departamento de Medicina Especializada da Unirio, Rio de Janeiro, RJ, Brasil

Endereço para correspondência:

Soniza Vieira Alves-Leon

Programa de Pós-Graduação em Neurologia da Unirio

Rua Mariz e Barros, 775 - Tijuca

CEP 20270-004 - Rio de Janeiro/RJ

E-mail: sonizavleon@globo.com

Recebido em: 23/6/08

Aceito em: 6/12/08 
bacterial sepsis and 17 had meconium aspiration syndrome. Acid-base imbalance occurred in $68.1 \%$ during the neonatal period. Lethality was $47.4 \%$ and occurred more often among boys $(65.6 \%)$ and in neonates of Afro-descendent mothers $(67.2 \%)$.

Conclusions: The incidence of neonatal epileptic seizures identified here was three to four times higher than the incidence found in hospitals of developed countries, although the cases characteristics were similar. Lethality rate was $47.4 \%$ and severe asphyxia was the most common pathological condition during delivery.

Key-words: infant, newborn; seizures; incidence; asphyxia neonatorum.

\section{Introdução}

O período neonatal é conceitualmente limitado às quatro primeiras semanas de vida ${ }^{(1)}$. A ocorrência de crises epilépticas no período neonatal pode ser o primeiro e único sinal de disfunção do sistema nervoso central (SNC) (2), sendo as manifestações neurológicas mais comuns nessa ocasião. As crises epilépticas nesse período podem ainda estar associadas a doenças sistêmicas. A identificação precoce das crises epilépticas no período neonatal bem como dos fatores de risco associados permite o desenvolvimento de estratégias com impacto na vida e sobrevida desses pacientes.

As crises epilépticas no período neonatal apresentam incidência variável na dependência dos critérios usados, se clínicos ou eletrencefalográficos ${ }^{(3)}$ : se apenas o EEG for considerado, esses registros corresponderão a menos de $50 \%$ da sua incidência real ${ }^{(3)}$.

As crises epilépticas no período neonatal possuem um padrão clínico distint, o que justifica a necessidade de classificação própria ${ }^{(4)}$. As crises neonatais compõem o Grupo III de classificação da International League Against Epilepsy (ILAE) de crises epilépticas autolimitadas: epilepsia neonatal familiar benigna (ENFB), encefalopatia mioclônica precoce (EMP) e síndrome de Otahara. Ainda no período neonatal, podem ocorrer as crises neonatais benignas e as crises febris ${ }^{(5)}$. A sua identificação nem sempre ocorre com facilidade em virtude da imaturidade do $\mathrm{SNC}^{(5)}$.

A maioria das crises neonatais é sintomática, com etiologia e distúrbios associados envolvendo um amplo espectro de doenças neonatais e disfunções metabólicas ${ }^{(4)}$, incluindo encefalopatia hipóxico-isquêmica, meningite, encefalite, hemorragia intracraniana, infartos cerebrais, anormalidades congênitas do cérebro, desordens metabólicas, erros inatos do metabolismo e desordens genéticas ${ }^{(4)}$.

O presente trabalho teve o objetivo de descrever a incidência de crises epilépticas no período neonatal, a frequência de óbitos e a presença de condições clínicas associadas durante as quatro primeiras semanas após o nascimento.

\section{Métodos}

A identificação dos pacientes de até quatro semanas de vida com crises epilépticas foi feita a partir de dados preliminares anotados nos registros da Disciplina de Pediatria da Universidade Federal do Estado do Rio de Janeiro (Unirio) após aprovação do Comitê de Ética em Pesquisa do Hospital Universitário Gaffée e Guinle. Os pacientes foram acompanhados até quatro semanas pelo Serviço de Neonatologia do Hospital Universitário Gaffrée e Guinle. O número total de nascidos vivos no período estudado foi obtido a partir dos livros de registro de partos do Centro Obstétrico. A confirmação e complementação de dados dos neonatos e respectivas mães foram obtidas a partir dos prontuários médico-hospitalares. Foram incluídas e analisadas informações de todos os neonatos que apresentaram uma ou mais crises epilépticas.

O diagnóstico de crises epilépticas foi feito por meio de critérios clínicos, considerando-se todas as crises ocorridas até a quarta semana de vida de nascidos vivos entre janeiro de 1995 e dezembro de 2004. O diagnóstico de crises epilépticas foi considerado quando houve registro de perda de consciência associada a manifestações motoras tônicas, clônicas ou tônico-clônicas simétricas ou assimétricas. Essas manifestações foram reunidas em um grupo denominado "crises epilépticas no período neonatal” (CEPN), independentemente da classificação proposta pela $\operatorname{ILAE}^{(6)}$, já que o objetivo do estudo foi determinar a incidência das CEPN e analisar as variáveis a elas associadas, levando-se em consideração que a classificação da ILAE requer um período maior de acompanhamento e a utilização de critérios eletrencefalográficos para classificar os tipos de epilepsia.

Trata-se de um estudo retrospectivo observacional de base hospitalar. A análise descritiva foi realizada por meio do programa Stata versão 8 , com tabelas, porcentagens e médias aritméticas acompanhadas dos respectivos desvios padrão $( \pm d p)$. Calculou-se a incidência de crises epilépticas no período neonatal na população de nascidos vivos durante o período estudado. Foram considerados também: incidência de neonatos com crises epilépticas a cada ano, tempo 
decorrido entre o nascimento e a primeira crise epiléptica e número de neonatos que evoluíram a óbito. As condições maternas estudadas foram: idade no momento do parto, cor (considerando a cor assumida no registro do prontuário), paridade, tabagismo e etilismo (conforme declaração da própria paciente), uso de drogas ilícitas por parte da mãe, infecções maternas (sífilis, toxoplasmose, rubéola, infecção pelo vírus da imunodeficiência adquirida), síndromes hipertensivas, diabetes melito, doenças neurológicas e psiquiátricas prévias ou durante a gestação. No período pré-natal, foram analisadas as variáveis relativas ao crescimento intrauterino restrito e ao sofrimento fetal crônico. Já no período intraparto, estudou-se: tipo de parto (vaginal, fórceps, cesáreo), tipo de anestesia, apresentação fetal, indicações do parto cesáreo, tempo de bolsa rota, características do líquido amniótico e boletim de Apgar.

As características dos neonatos com CEPN incluíram: gênero, recém-nascido único ou gemelar, idade gestacional, peso ao nascer e peso versus idade gestacional. As condições patológicas dos neonatos analisadas foram: infecções (congênitas e adquiridas) definidas por critérios clínicos e laboratoriais, desordens metabólicas (hipo e hiperglicemia e acidose), icterícia, anemia, síndrome de membrana hialina, síndrome de aspiração meconial e outras doenças, como malformações e doenças do sistema nervoso. As variáveis epidemiológicas e demográficas foram classificadas de acordo com as informações registradas no prontuário médico e as varáveis laboratoriais foram classificadas de acordo com os valores de referência utilizados pelo hospital.

\section{Resultados}

O número de nascidos vivos, no período de janeiro de 1995 a dezembro de 2004, foi 6.600, em um total de 6.483 partos. Nesses dez anos, foram observados 61 casos de CEPN, o equivalente a uma incidência de crises epilépticas de 9,2 por mil neonatos (IC95\%: 7,1-11,9). Considerando-se o número de neonatos ano a ano, a incidência de neonatos com crises epilépticas na população estudada sofreu importantes variações, oscilando entre 1,6 e 16,6 por mil nascidos vivos (Figura 1).

A primeira crise epiléptica ocorreu nas primeiras 12 horas de vida em 24 (45,3\%) casos, seguida pela ocorrência entre 13 e 72 horas após o nascimento em $17(32,1 \%)$ pacientes e, acima desse período, em 12 (22,6\%), não sendo possível identificar o tempo de aparecimento das convulsões em oito casos.

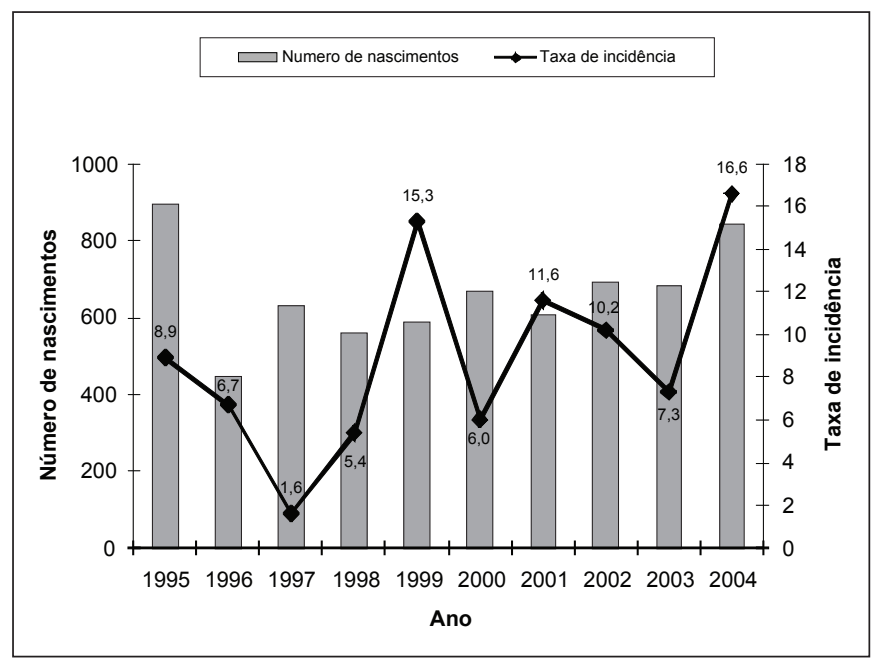

Figura 1 - Incidência de crises epilépticas por mil neonatos e número de nascimentos por ano, de janeiro de 1995 a dezembro de 2004, HUGG/Unirio.

A idade materna dos neonatos que apresentaram convulsões variou entre 14 e 42 anos (média 27,8 7,8), prevalecendo a faixa entre 30 e 39 anos ( $41 \%)$. Houve predomínio de afro-descendentes com $41(67,2 \%)$ casos, seguido pelas mães de cor branca $(32,8 \%)$. A maioria era primípara $(36,1 \%)$, seguidas das secundíparas (24,6\%). Multíparas somaram $39,3 \%$. Todas deram informações acerca de tabagismo e etilismo, com registro de $11(18 \%)$ fumantes e três $(4,9 \%)$ usuárias regulares de bebida alcoólica. Apenas uma $(1,7 \%)$ gestante dentre 59 pacientes revelou uso de drogas ilícitas e, em dois casos, não havia relato no prontuário.

Todas as mães realizaram sorologia para sífilis, com resultado positivo em três $(4,9 \%)$ : duas crianças receberam alta após tratamento específico, enquanto uma terceira evoluiu para óbito por complicações metabólicas e respiratórias múltiplas. A pesquisa de $\operatorname{IgM}$ para toxoplasmose foi realizado em 37 mães, com resultado positivo em duas (5,4\%); uma delas recebeu tratamento específico durante a gestação, que resultou em neonato sem toxoplasmose congênita e a outra deu à luz neonato com neurotoxoplasmose. Nenhuma das 36 gestantes testadas teve IgM positiva para rubéola e 41 mulheres realizaram sorologia anti-HIV 1 e 2 e, em apenas um caso, houve positividade.

Foram registradas $19(32,8 \%)$ pacientes com síndromes hipertensivas durante a gestação ou na ocasião do parto. Foram identificados nove $(15,5 \%)$ casos de diabetes melito (gestacional ou pré-existente) dentre as 58 mães de neonatos com CEPN. Não houve informações quanto à glicemia em três pacientes. $\mathrm{O}$ crescimento intrauterino restrito ocorreu 
em 25,9\% dos 58 casos analisados. O sofrimento fetal crônico esteve presente em $34,5 \%$ desses 58 neonatos. Houve perda de informação em três casos.

Em 38 (62,3\%) pacientes com CEPN, o parto ocorreu por cesariana, enquanto apenas $6,6 \%$ dos neonatos foram retirados a fórceps. Dos $19(31,1 \%)$ partos vaginais, 14 foram em apresentação cefálica e cinco, pélvica. Inúmeras causas estiveram presentes na indicação da via do parto operatório (cesáreo ou a fórceps), muitas delas associadas em uma só criança. O sofrimento fetal agudo foi a causa mais frequente $(45,9 \%)$ de indicação de cesariana entre os neonatos com CEPN, seguido da amniorrexe prematura (24,3\%). Em três casos, a cesariana foi indicada por pós-maturidade associada ao sofrimento fetal agudo, à pré-eclampsia grave e à macrossomia fetal e iteratividade. $\mathrm{O}$ trabalho de parto prematuro ocorreu em três casos com indicação de cesariana, sempre associado a outras condições que motivaram o parto por via alta. Em sete pacientes, síndromes hipertensivas estiveram presentes, todas associadas a outras condições de indicação da cesariana. Como representação máxima da

Tabela 1 - Características dos recém-nascidos com crises epiléticas no período neonatal

\begin{tabular}{|c|c|c|}
\hline & $\mathrm{n}$ & $\%$ \\
\hline \multicolumn{3}{|l|}{ Número de fetos $(n=61)$} \\
\hline Feto único & 56 & 91,8 \\
\hline Primeiro gemelar & 3 & 4,9 \\
\hline Segundo gemelar & 2 & 3,3 \\
\hline \multicolumn{3}{|l|}{ Apgar $1^{\circ}$ minuto $(n=57)$} \\
\hline Até 3 & 29 & 50,9 \\
\hline$>3$ & 28 & 49,1 \\
\hline \multicolumn{3}{|l|}{ Apgar $5^{\circ}$ minuto $(n=61)$} \\
\hline Até 7 & 41 & 67,2 \\
\hline$>7$ & 20 & 32,8 \\
\hline \multicolumn{3}{|c|}{ Idade gestacional em semanas $(n=61)$} \\
\hline$<30$ & 7 & 11,5 \\
\hline 30 a 36,9 & 25 & 41,0 \\
\hline 37 a 41,9 & 24 & 39,3 \\
\hline$\geq 42$ semanas & 5 & 8,2 \\
\hline \multicolumn{3}{|l|}{ Peso ao nascer em gramas $(n=61)$} \\
\hline Baixo peso $(\mathrm{PN}<2500 \mathrm{~g})$ & 31 & 50,8 \\
\hline Muito baixo peso $(\mathrm{PN}<1500 \mathrm{~g})$ & 14 & 23,0 \\
\hline Extremo baixo peso $(\mathrm{PN}<1000 \mathrm{~g})$ & 4 & 6,6 \\
\hline \multicolumn{3}{|l|}{$\begin{array}{l}\text { Adequação de peso versus idade } \\
\text { gestacional }(n=61)\end{array}$} \\
\hline PIG & 31 & 50,8 \\
\hline AIG & 23 & 37,7 \\
\hline GIG & 7 & 11,5 \\
\hline
\end{tabular}

PN: peso ao nascer doença hipertensiva, houve um caso da síndrome HELLP (Hemolysis; Elevated Liver Enzimes; Low Platelets). Houve quatro gestações gemelares que terminaram em cesariana sem que essa fosse a indicação principal da cirurgia. A aplicação do fórceps foi indicada em três casos para abreviar o tempo prolongado do período expulsivo e, no quarto, por adinamia uterina e amniorrexe.

O tempo de bolsa rota maior do que 12 horas apresentouse em $16(26,7 \%)$ casos dentre os 57 neonatos para os quais essa informação estava disponível. O ologoâmnio esteve presente em 18 casos e em uma gestação foi observado plidrâmnio. A presença de líquido amniótico meconial espesso foi observada em sete $(11,5 \%)$ casos e mecônio fluído, em quatro casos. Dentre esses 61 recém-nascidos, a idade gestacional variou de 24,1 a 42,1 semanas (mediana 36,3) e o peso ao nascer de 525 a $4760 \mathrm{~g}$ (mediana $2455 \mathrm{~g}$ ). Entre os casos de CEPN, 91,8\% eram fetos únicos, 4,9\% eram primeiro gemelares e 3,3\%, segundo gemelares. A maioria dos 61 recém-nascidos era do sexo masculino $(65,6 \%)$. As demais características demográficas dos neonatos que apresentaram CEPN apresentam-se na Tabela 1.

Dentre 49 casos documentados, $32(65,3 \%)$ apresentaram sepse de causa bacteriana em diferentes fases da vida neonatal, relacionada ou não à ruptura de membranas ou à prematuridade. Em 12 casos, não houve informação suficiente para caracterizar ou afastar a sepse neonatal. Desordens metabólicas envolvendo hipoglicemia e hiperglicemia estiveram presentes, respectivamente, em $26(56,5 \%)$ e três $(6,5 \%)$ dos 46 neonatos com tais dados disponíveis. Dentre 47 neonatos com o dado disponível, $32(68,1 \%)$ apresentaram algum grau de acidose metabólica até 28 dias de vida. Houve relato de icterícia neonatal precoce ou tardia em $52,1 \%$ dos casos analisados. Porém, em nenhum deles confirmou-se incompatibilidade sanguínea $\mathrm{ABO}$ ou pelo fator Rh e, em nenhum caso de icterícia neonatal, a concentração de bilirrubina foi $>20 \mathrm{mg} / \mathrm{dL}$. Dentre os 53 recém-nascidos com dados disponíveis, dez $(18,9 \%)$ desenvolveram síndrome de membrana hialina e $17(32,1 \%)$ apresentaram síndrome de aspiração meconial. Além disso, observaram-se 17 casos de malformações. A letalidade foi de $27(44,0 \%)$ casos.

\section{Discussão}

A morbidade neurológica consequente a condições desfavoráveis no pré-natal, durante o trabalho de parto, no parto propriamente dito ou no pós-parto é fato relevante e, por vezes, é negligenciado nas Unidades de Saúde. O presente 
estudo analisou os registros sistemáticos das variáveis pertinentes durante o ciclo grávido-puerperal feitos por obstetras e neonatologistas do Hospital Universitário Gaffée e Guinle com o objetivo de contribuir para um conhecimento melhor das crises epilépticas neonatais, sua evolução e fatores presentes na série de casos analisada de forma descritiva.

Neste estudo, a incidência de crises epilépticas neonatais foi de 9,2 por mil neonatos nos dez anos de registros analisados. Lanska e Lanska encontraram, em hospitais norte-americanos, uma incidência de 2,84 para cada mil nascidos vivos em um período de $12 \operatorname{anos}^{(7)}$, enquanto Gunn e Cable ${ }^{(8)}$ encontraram 2,33 casos em mil nascidos vivos na Nova Zelândia após quatro anos de seguimento. Comparativamente, nossos dados apontam uma frequência de crises epilépticas neonatais quase 3,5 vezes maior do que a encontrada na população americana e quatro vezes maior do que a observada na Nova Zelândia. Além disso, outros estudos baseados em dados de registros médicohospitalares encontraram taxas 2,6 a 4,6 vezes inferiores às do presente estudo, sendo 3,5 por mil nascidos vivos em Kentucky ${ }^{(9)}$ e 2 a 2,5 por mil em Tóquio. Nesse último estudo, referente a um período de dez anos, Kawakami et al mostraram uma redução de 2,5 para 2,0 por mil nos cinco últimos anos, o que foi creditado à melhoria da assistência ao parto e dos cuidados na Unidade de Terapia Intensiva neonatal da instituição ${ }^{(10)}$. Tais dados reforçam a ideia da ampla variabilidade na incidência (especialmente em se tratando de diferentes desenhos de estudos, sejam eles de base populacional ou de pacientes admitidos em unidades neonatais de terapia intensiva), com diversos fatores de risco e etiológicos analisados e, sobretudo, critérios diagnósticos inconsistentes, levando a uma potencial confusão com comportamentos não epilépticos. Além disso, devem-se considerar condições socais diferentes de cada região, visto que a principal causa de crises epilépticas neonatais é a asfixia neonatal, que se associa às condições de atendimento à gestante no período pré-natal e durante o parto.

Quase metade das crises epiléticas ocorreu nas primeiras 12 horas de vida. A evolução para o óbito, em nossa casuística, foi $44 \%$, semelhante ao encontrado por Asindi et al (50\%) em unidade neonatal de hospital universitário nigeriano ${ }^{(11)}$. Por outro lado, a taxa de letalidade observada no presente estudo foi cerca de 2,5 vezes superior àquela encontrada por Airede (19,3\%) e quase duas vezes maior que Alcover-Block et al $(27,7 \%)$ e Brankovic et al $(25,4 \%)^{(12-14)}$. Brankovic et al chamam atenção para o decréscimo significativo na mortalidade associada às crises epilépticas neonatais de ocorrência tardia (após 12 horas do nascimento) ${ }^{(14)}$. Considerando esse aspecto, o alto índice de letalidade observado pode, talvez, ser atribuído ao fato de que quase metade dos neonatos do presente estudo sofreu crises epilépticas nas primeiras 12 horas após o nascimento.

Os resultados mostram, ainda, maior frequência de mães entre 30 e 39 anos de idade (41\%), principalmente afrodescendentes $(67,2 \%)$. Saliba et al ${ }^{(15)}$ encontraram idade materna mais baixa, entre 18 e 24 anos, como fator de risco significativo para crises epilépticas neonatais e não observaram qualquer diferença étnica. Lanska e Lanska ${ }^{(7)}$ também não encontraram diferença significativa de risco ao comparar os grupos raciais e o gênero, obtendo-se resultados semelhantes aos de Lanska et al ${ }^{(16)}$. Isso mostra que não há consenso sobre a contribuição idade da mãe e grupos raciais para o desenvolvimento de crises epilépticas neonatais.

Foram identificadas 19 gestantes $(32,8 \%)$ com síndromes hipertensivas durante a gestação ou por ocasião do parto na presente casuística. Garcias da Silva et a $l^{(1)}$ encontraram apenas $13,5 \%$ de bebês portadores de crises epilépticas neonatais cujas mães haviam apresentado doença hipertensiva específica da gravidez. Kaye ${ }^{(17)}$ relata que a presença de pré-eclâmpsia ou eclâmpsia aumentou em mais de dez vezes o risco de asfixia neonatal (OR: 10,6; IC95\%: 2,9-38,5).

O presente estudo mostrou, ainda, que $50,8 \%$ dos neonatos com crises epilépticas neonatais apresentaram peso ao nascer inferior a $2500 \mathrm{~g}$, o que está de acordo com dados de outros estudos que apontam o baixo-peso como um dos fatores etiológicos mais frequentemente associados a essa condiçãa ${ }^{(7,10,14-16)}$. Alterações metabólicas e infecciosas também estiveram presentes nos neonatos com a condição convulsiva.

Por fim, mesmo tratando-se de unidade hospitalar de referência para o atendimento de situações especiais, como soropositividade para o HIV e síndromes hipertensivas maternas, os dados indicam frequência de crises epilépticas neonatais maior do que a observada em outros estudos, o que sugere a necessidade de mudar os padrões de atendimento à gestante e ao neonato.

Finalmente, as crises epilépticas neonatais ocorreram, em sua maioria, antes de 12 horas de vida e em recém-nascidos com baixo índice de Apgar. Medidas como a reanimação adequada na sala de parto, os cuidados nas primeiras horas de vida, a inclusão de neuropediatras nas equipes multiprofissionais e a implementação de melhorias na qualidade no atendimento às gestantes podem modificar o quadro descrito no presente estudo. 


\section{Referências bibliográficas}

1. Garcias da Silva LF, Nunes ML, da Costa JC. Risk factors for developing epilepsy after neonatal seizures. Pediatr Neurol 2004;30:271-7.

2. Engel J Jr, Pedley TA. Epilepsy: a comprehensive textbook. Philadelphia: Lippincott-Raven; 1997.

3. Scher MS. Neonatal seizure classification: a fetal perspective concerning childhood epilepsy. Epilepsy Res 2006;70 (Suppl 1):S41-57.

4. da Costa JC, Nunes ML, Fiori RM. Convulsões no período neonatal. J Pediatr (Rio J) 2001;77 (Suppl 1):S115-22

5. Engel J Jr. Report of the ILAE classification core group. Epilepsia 2006;47: 1558-68.

6. Tan K, Dear PR, Newell SJ. Clinical decision support systems for neonatal care. Cochrane Database Syst Rev 2005:CD004211.

7. Lanska MJ, Lanska DJ. Neonatal seizures in the United States: results of the National Hospital Discharge Survey, 1980-1991. Neuroepidemiology 1996;15:117-25.

8. Gunn T, Cable G. Neonatal seizures: aetiology and outcome. N Z Med J 1984;97:898-900.

9. Lanska MJ, Lanska DJ, Baumann RJ. A population-based study of neonatal seizures in Fayette County, Kentucky: comparison of ascertainment using different health data systems. Neuroepidemiology 1995;14: 278-85.
10. Kawakami T, Yoda $\mathrm{H}$, Shima $Y$, Akamatsu $\mathrm{H}$. Incidence and causes of neonatal seizures in the last 10 years (1981-1990). No To Hattatsu 1992;24:525-9.

11. Asindi AA, Antia-Obong OE, Ibia EO, Udo JJ. Neonatal seizures in Nigerian infants. Afr J Med Med Sci 1995;24:243-8.

12. Airede KI. Neonatal seizures and a 2-year neurological outcome. J Trop Pediatr 1991;37:313-7.

13. Alcover-Bloch E, Campistol J, Iriondo-Sanz M. Neonatal seizures, our experience. Rev Neurol 2004;38:808-12.

14. Branković D, Jovanović R, Karović D, Sindjić V, Milenković-Vucić M, Kovacević V. Convulsions in neonates and early neonatal mortality. Jugosl Ginekol Perinatol 1990;30:161-4.

15. Saliba RM, Annegers FJ, Waller DK, Tyson JE, Mizrahi EM. Risk factors for neonatal seizures: a population-based study, Harris County, Texas, 1992-1994. Am J Epidemiol 2001;154:14-20.

16. Lanska MJ, Lanska DJ, Baumann RJ, Kryscio RJ. A population-based study of neonatal seizures in Fayette County, Kentucky. Neurology 1995;45:724-32.

17. Kaye D. Antenatal and intrapartum risk factors for birth asphyxia among emergency obstetric referrals in Mulago Hospital, Kampala, Uganda. East Afr Med J 2003;80:140-3.

18. Low JJ, Yeo GS. Eclampsia: are we doing enough? Singapore Med J 1995;36:505-9. 